\title{
Clinicopathological correlation of deep retinal vascular anomalous complex in age related macular degeneration
}

\author{
B A Lafaut, S Aisenbrey, C Vanden Broecke, K U Bartz-Schmidt
}

\begin{abstract}
Aims-To analyse the histopathology of "deep retinal vascular anomalous complex" or "chorioretinal anastomosis". Methods-Six patients with a deep retinal vascular anomalous complex (age range 66-88 years) had fundus photography and fluorescein angiography not more than 14 days before foveal translocation surgery. Four patients were also documented with indocyanine green angiography. The surgical specimens were serially sectioned and stained in a stepped fashion with Masson trichrome, periodic acid Schiff, and phosphotungstic acid haematoxylin, a histochemical stain for fibrin.
\end{abstract}

Results-A subretinal fibrovascular membrane was surrounded by a rim consisting of diffuse drusen (basal laminar deposits), retinal pigment epithelium, and amorphous, fibrinous material interspersed with remains of outer segments in all specimens. In two specimens vascular structures were identified that left the specimen towards the retina. Amorphous material with the remains of outer segments was not found on the retinal side of the fibrovascular tissue itself but in four specimens a small neuroretinal portion (outer nuclear layer) was adherent to the complex. In three specimens a thin fibrocellular membrane was seen at the choroidal side of the diffuse drusen.

Conclusion-Deep retinal vascular anomalous complex represents histologically neovascularisation growing out of the neuroretina, into the subretinal space, which mimics choroidal neovascularisation. The term therefore appears rightly chosen.

(Br f Ophthalmol 2000;84:1269-1274)

Age related macular degeneration is the leading cause of blindness in elderly people throughout the Western world. ${ }^{12}$ The ophthalmoscopic marker of the disorder is the presence of macular soft drusen and pigmentary changes. ${ }^{3}$ Non-neovascular age related macular degeneration usually has little impact on visual acuity unless chorioretinal atrophy develops. Neovascular age related macular degeneration is the major cause of severe and irreversible vision loss in age related macular degeneration. ${ }^{4}$ The neovascular disease is due to the growth of new blood vessels from the choroid in Bruch's membrane ${ }^{56}$ and in the subretinal space. ${ }^{78}$ If left untreated, eventually a large fibrovascular scar, a so called disciform scar, will replace the retinal pigment epithelium and the photoreceptors. ${ }^{6} 9$ In a few end stage disciform scars, retinal choroidal vascular anastomosis or retinal vessels that contribute to the vascularisation of the scars have been identified funduscopically, angiographically, and histopathologically..$^{6910}$

In 1992, Hartnett et $a l^{11}$ recognised age related pigment epithelial detachments associated with a peculiar retinal vascular abnormality in the absence of disciform scarring. Fluorescein angiography revealed a feeding retinal arteriole dipping towards the retinal pigment epithelium, forming "an angiomatous lesion" in the subretinal space. Kuhn et al ${ }^{12}$ described similar lesions by fluorescein and indocyanine green angiography and termed such lesions "chorioretinal anastomosis". Such lesions, "deep retinal vascular anomalous complex" or "chorioretinal anastomosis", have also since been identified in the absence of a pigment epithelial detachment. ${ }^{13}{ }^{14}$ This entity is recognised as a cause of severe visual loss in patients with age related macular degeneration. The outcome after laser photocoagulation is poor, even when located extrafoveally. ${ }^{12}$ So far no clinicopathological correlations are available of such lesions.

Recently, surgical specimens have become available as a new interest has developed in the surgical removal of choroidal neovascularisation in age related macular degeneration with translocation of the fovea towards an area with healthier retinal pigment epithelium. We have analysed the histological appearance of surgically removed submacular tissue in six eyes with a deep retinal vascular anomalous complex with an associated pigment epithelial detachment. We report the histoarchitecture of these specimens, which may contribute to a better understanding of this peculiar complication of age related macular degeneration.

\section{Materials and methods}

STUDY POPULATION

Six patients with a deep retinal vascular anomalous complex or chorioretinal anastomosis (age range 66-88 years) had fundus photography and fluorescein angiography not more than 14 days before foveal translocation surgery. Four patients were also documented with indocyanine green angiography. The fluorescein angiogram was obtained after intravenous injection of $5 \mathrm{ml}$ sodium fluorescein $10 \%$ (Alcon Pharma GmbH, Freiburg, Germany) with a Canon fundus camera (Canon Inc, Neuss, Germany) linked to a Kodak high resolution camera (OIS, Ophthalmic Imaging 
Systems, Polytech Ophthalmologie $\mathrm{GmbH}$, Rossdorf, Germany). Pictures were taken up to 10 minutes after intravenous injection. The indocyanine green angiogram was obtained after intravenous injection of $50 \mathrm{mg}$ indocyanine green (Pulsion Medical Systems, Munich, Germany) dissolved in $10 \mathrm{ml}$ with the same camera system. Pictures were taken up to 30 minutes after intravenous injection. None of the patients had diseases predisposing to choroidal neovascularisation other than age related macular degeneration, such as high myopia, multifocal choroiditis, or angioid streaks.

HISTOLOGICAL ANALYSIS

The specimens were fixed in $10 \%$ neutrally buffered formalin, dehydrated, and embedded

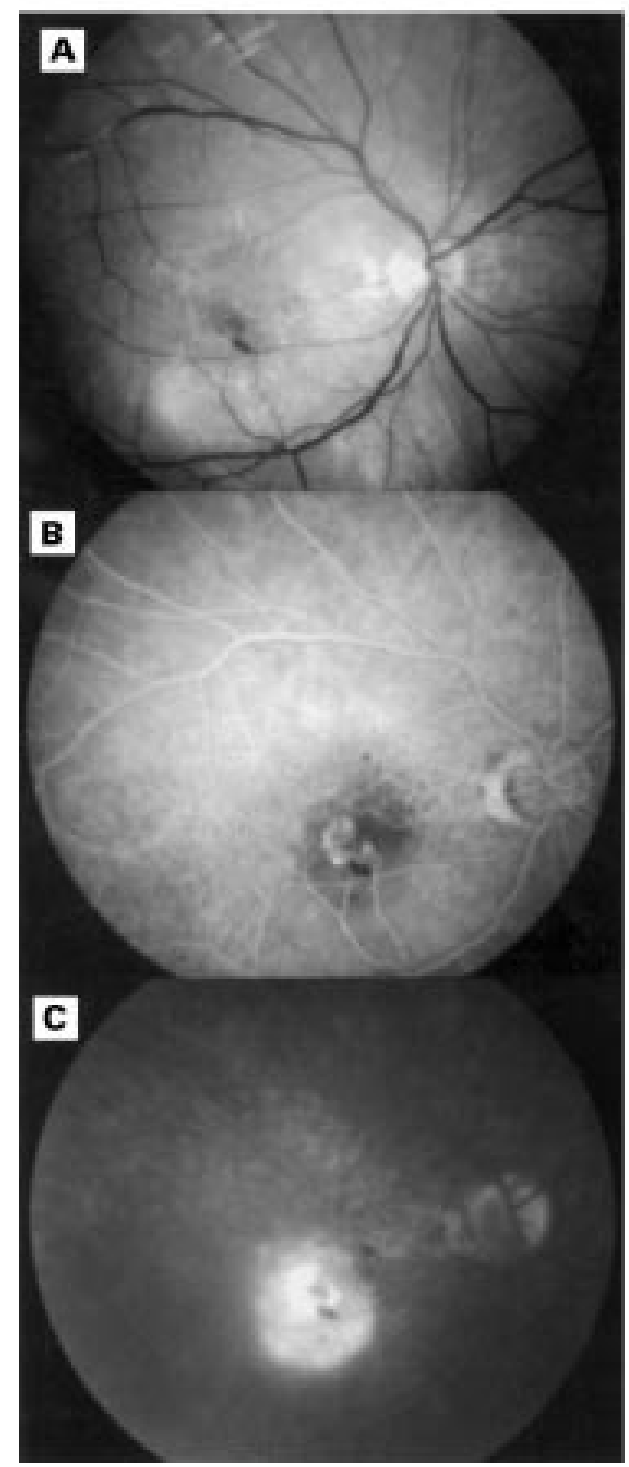

in paraffin for light microscopy. The membranes were serially sectioned and stained in a stepped fashion with haematoxylin and eosin, Masson trichrome (MTC), and periodic acid Schiff (PAS). Multiple sections of each membrane were stained with phosphotungstic acid haematoxylin histochemical stain for fibrin. The presence of lipofuscin was demonstrated by autofluorescence microscopy on PAS stained sections.

\section{Results}

CLINICAL EXAMINATION

A pigment epithelial detachment, intraretinal haemorrhages, and retinal exudates were observed preoperatively in all six study eyes (Figs 1A-D, 2A). Subconfluent soft drusen were

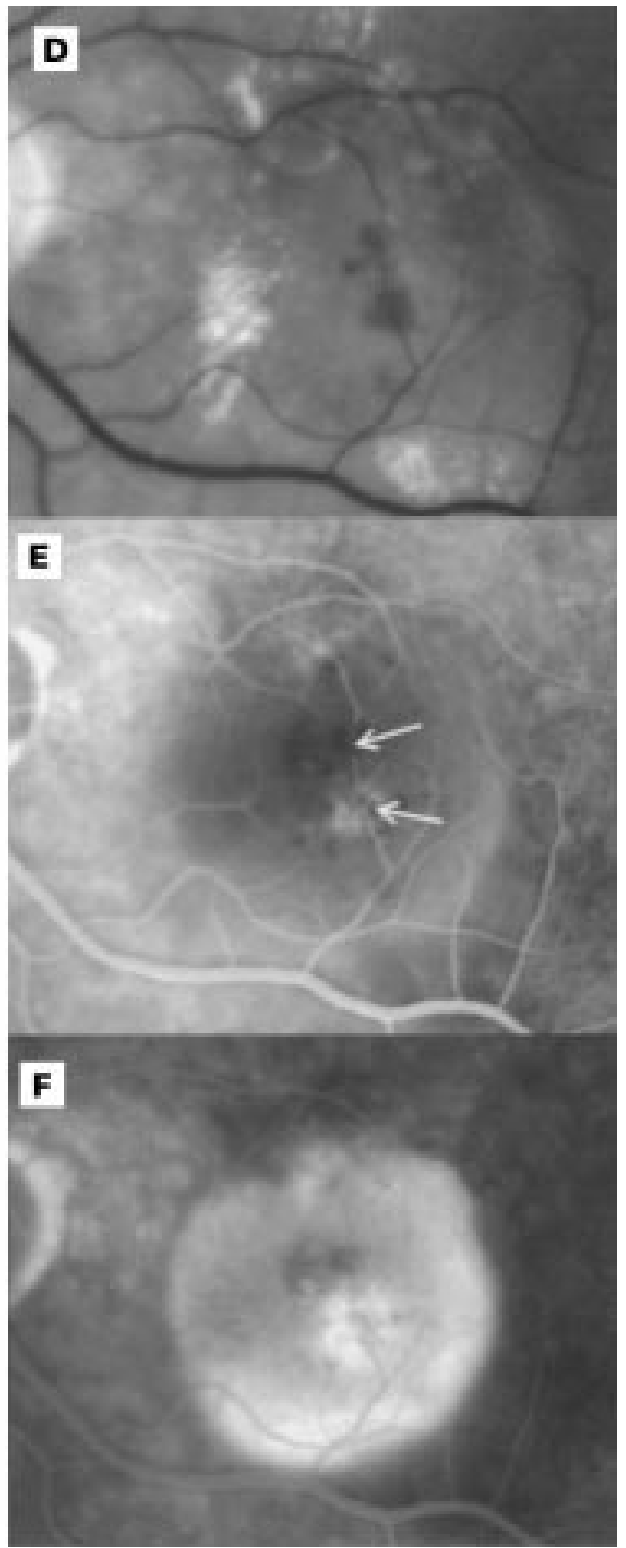

Figure 1 (A), (B), and (C) respectively red-free, venous, and late phase fluorescein angiography of a 71 year old patient. Intraretinal lipid exudates and haemorrhages are seen on the red-free photograph. A subretinal neovascular net is seen in the right eye which appears connected to at least two retinal vessels in the early phase. The hyperfluorescence of this deep retinal vascular anomalous complex persists in the late phase. The associated retinal pigment epithelial detachment appears hypofluorescent in the early phase but becomes hyperfluorescent in the late phase. (D), (E), and $(F)$ respectively enlarged red-free, venous, and late phase fluorescein angiography of a 70 year old patient. Intraretinal lipid exudates and

haemorrhages are seen, arrows indicate retinal vessels that appear to dip into the deep retinal vascular anomalous complex. The associated serous pigment epithelial detachment is clearly demonstrated in the late phase. 
found in five of six. Fluorescein angiography identified a small hyperfluorescent lesion from the early phase of the angiogram (Figs 1A, C, $2 \mathrm{~B}, \mathrm{C})$, that appeared to be fed by one or more serous pigment epithelial detachment was outlined by its hyperfluorescence (Fig 1C, D). Indocyanine green angiography showed either a small vascular net (Fig 2E) or only a focal hyperfluorescence in the early phase. The hot spot persisted in the late phase, lying within a hypofluorescent pigment epithelial detachment (Fig 2F). The vascular anomaly was not located subfoveally (juxtafoveally in two eyes, extrafoveally in four eyes) but the associated pigment epithelial detachment had undermined the entire fovea. Subconfluent soft drusen were observed in four fellow eyes. A macula of three fellow eyes. In two of these a large calibre retinal choroidal anastomosis was observed. In one fellow eye, a pigment epithe-

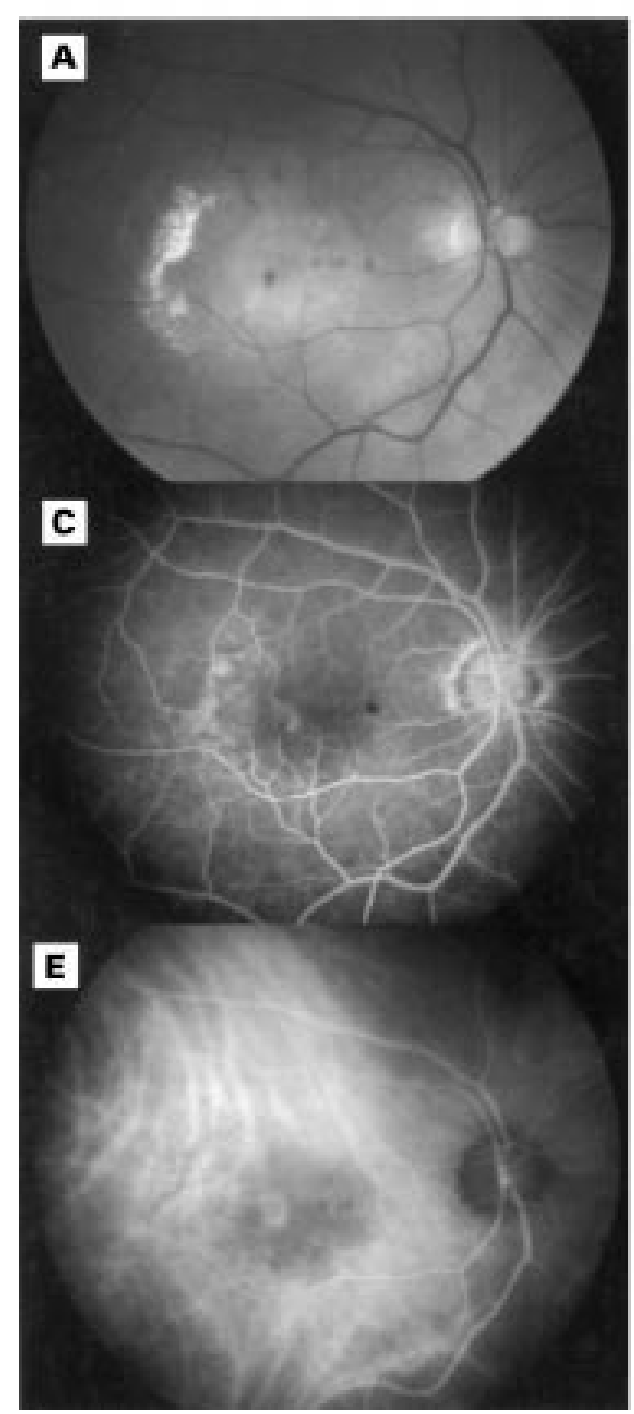
retinal vessels. In the late phase the associated rather small disciform scar was seen in the

lial detachment, intraretinal haemorrhages, and retinal exudates were found to be associated with the presence of a deep retinal vascular anomalous complex.

\section{PATHOLOGICAL EXAMINATION}

A subretinal fibrovascular membrane was surrounded by a rim consisting of diffuse drusen (basal laminar deposits), retinal pigment epithelium, and amorphous, fibrinous material interspersed with remains of outer segments in all specimens (Fig 3A, C). A monolayer of retinal pigment epithelial cells was seen in the surrounding rim but only very few retinal pigment epithelial cells were found between the fibrovascular tissue and the diffuse drusen (Fig 3A-D). Scattered pigmented cells were found within the fibrovascular tissue. In two specimens vascular structures were identified that left the specimen towards the retina (Fig 3A, B). Amorphous material with remains of outer segments was not found on the retinal side of the fibrovascular

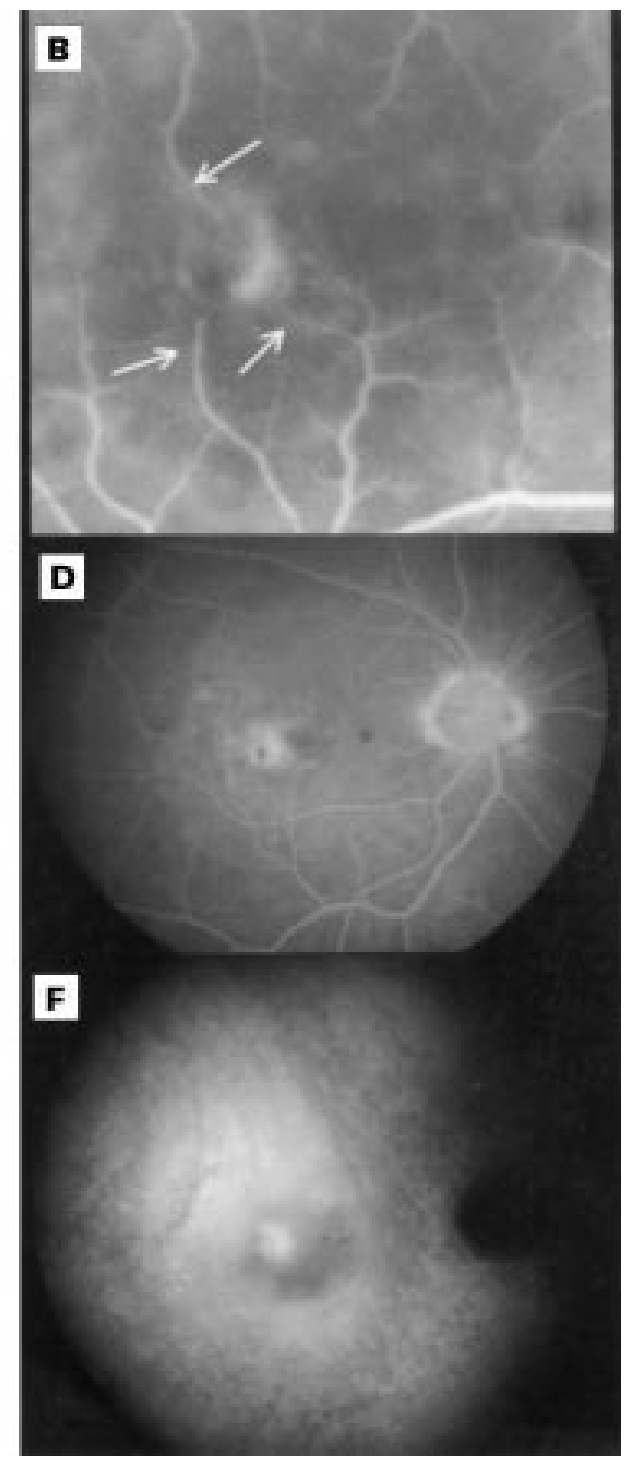

Figure 2 Red-free $(A)$ fluorescein angiogram $(B, C$, and $D)$ and indocyanine green angiogram of a 73 year old patient. On the red-free photograph, intraretinal lipid exudates, haemorrhages, and a drusenoid pigment epithelial detachment can be seen. Fluorescein angiography indicates a deep retinal vascular anomalous complex that appears to be connected to three retinal vessels (arrows). The venous $(E)$ and late phase $(F)$ indocyanine green angiogram identifies a vascular net. The lesion persists as a hot spot in the late phase, entirely within a hypofluorescent zone corresponding to the retinal pigment epithelial detachment. 
tissue itself but in four specimens a small neuroretinal portion (predominantly outer nuclear layer) was adherent to the complex. In three specimens a smaller, fibrocellular component was seen at the choroidal side of the diffuse drusen and therefore within the Bruch's membrane (Fig 3C, D, E). A defect in the diffuse drusen was found in two of the latter specimens with the edges curling towards the choroid (Fig $3 \mathrm{E})$ as if tissue had grown from the subretinal space into the Bruch's membrane, at the choroidal side of the diffuse drusen. Some melanolipofuscin bearing cells were scattered within the intra-Bruch's fibrocellular component. In three specimens, pale staining, fine granular material was observed at the choroidal side of the diffuse drusen (Fig 3F).

\section{Discussion}

In this study, we determined the histopathological features of deep retinal vascular anomalous complex in age related macular degeneration, which is typically associated with retinal lipid exudation, intraretinal haemorrhages, subconfluent drusen, and a shallow detachment of the retinal pigment epithelium. ${ }^{11-14}$ Many authors have reported the histological characteristics of choroidal neovascularisation
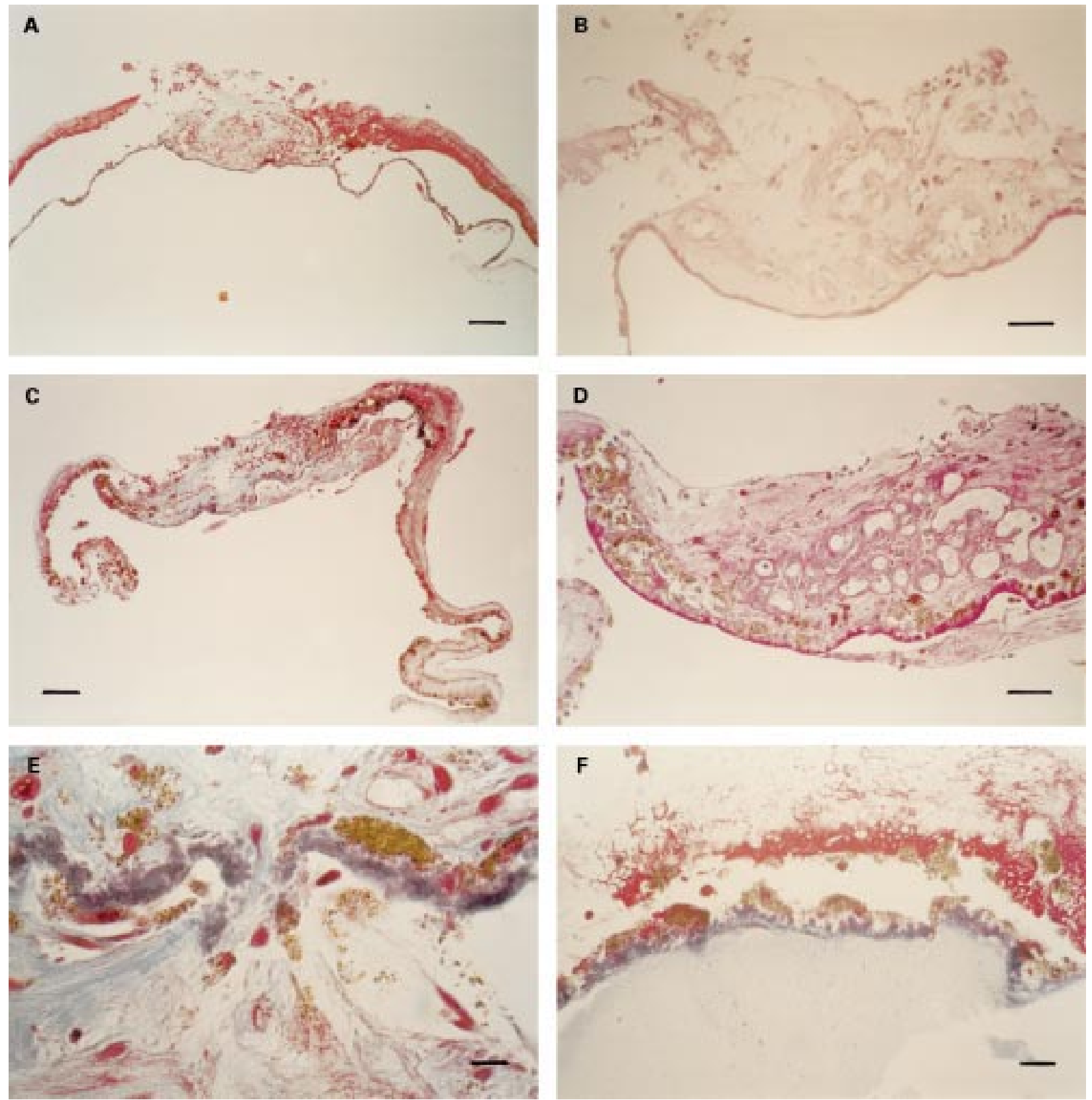

Figure 3 (A) MTC stain, bar $=100 \mu \mathrm{m}$, overview of specimen corresponding to Figure 2. A subretinal central fibrovascular nodule is seen surrounded by a rim composed of amorphous material, retinal pigment epithelium, and diffuse drusen. (B) PAS stain, bar $=50$ um, detail of same specimen as $(A)$. The fibrovascular tissue immediately contacts the diffuse drusen. Some vessels leave the specimen towards the retina. (C) MTC stain, bar $=100 \mu m$, overview of specimen corresponding to Figure $1 A, B, C$. A central fibrovascular nodule is seen, consisting both of a subretinal vascular component and an intra-Bruch's fibrocellular component, with surrounding rim. (D) PAS stain, bar $=50 \mu \mathrm{m}$, detail of same specimen as (C). A rich vasculature is demonstrated in the subretinal part of the specimen. (E) MTC stain, bar $10 \mu \mathrm{m}$, detail of same specimen as (C). An interruption of the diffuse drusen layer is seen with a fibrous tissue connecting the subretinal with the intra-Bruch's component. (F) MTC stain, bar 25 um, detail of a third specimen, at the level of the rim. Pale staining, fine granular material is seen at the choroidal side of the diffuse drusen. 
in age related macular degeneration ${ }^{5-8}$ but the pathological correlate of deep retinal vascular anomalous complex has not yet been reported. Choroidal neovascularisation in age related macular degeneration corresponds to fibrovascular tissue growing from the choroid primarily into the Bruch's membrane ${ }^{56}$ but also subretinally. ${ }^{78}$ We have used light microscopy for this study as the major cellular components (retinal pigment epithelium, vascular endothelium, fibrocytes, macrophages, and photoreceptors) as well as extracellular components (collagen, diffuse drusen, and fibrin) can be correctly identified by light microscopy alone. ${ }^{15}$ Diffuse drusen is a characteristic histological manifestation of age related macular degeneration and corresponds to the electron microscopic term basal laminar deposits. ${ }^{5}{ }^{6}$ It is a granular, PAS positive deposition that stains metachromatically blue purple on MTC, seen at the level of the basement membrane of the retinal pigment epithelium or between the retinal pigment epithelium and the remainder of Bruch's membrane. It can be differentiated from the outer Bruch's membrane since the latter is a homogeneous linear PAS positive layer.

We have found a small subretinal fibrovascular membrane to correspond to the deep retinal vascular anomalous complex in the six specimens examined. Relatively large vessels could be traced to leave the fibrovascular membrane towards the neuroretina in two specimens. Even when surrounding soft drusen were clinically documented in five eyes, their pathological counterpart-pale staining fine granular material at the choroidal side of the diffuse drusen, was only histologically identified in three specimens. Either this material remained adherent to the Bruch's membrane at the time of surgery or, being fragile, it was lost when the specimen was removed through the sclerotomy. In four specimens some outer nuclear layer neuroretinal tissue was adherent to the fibrovascular membrane, suggesting that the fibrovascular tissue is closely adherent to the neuroretina. Furthermore, these membranes had to be peeled from the neuroretina as they remained adherent to the neuroretina when the retina was iatrogenically detached from the choroid by injection of subretinal balanced salt solution at the time of surgery. This contrasts with classic or occult choroidal neovascular membranes that tend to remain adherent to the choroid when the overlying retina is detached during the surgical procedure. Another argument that the removed fibrovascular membrane is supplied by the retina is an indirect one. For the three membranes that consisted of a bilayer, a subretinal, and an intra-Bruch's component, only the subretinal component was vascularised. The intra-Bruch's fibrocellular component appeared to have originated from the subretinal space as melanolipofuscin bearing cells which must have migrated from the subretinal space were incorporated in its stroma. Despite this circumstantial evidence, choroidal vascular anastomoses might have been overlooked and the choroidal vascular contribution to these lesions can not be excluded with certainty.

Subretinal neovascularisation from the retina has been previously reported in rodents. When the outer nuclear layer had disappeared in RCS dystrophic animals, ${ }^{16}$ after light toxicity ${ }^{17}$ or pharmacological toxicity, ${ }^{18}{ }^{19}$ retinal capillaries may come into contact with the retinal pigment epithelium and with time the capillaries proliferate and are covered by the retinal pigment epithelium. In deep retinal vascular anomalous complex a rather similar process may occur. The major difference however is that the new vessels are not embedded by retinal pigment epithelium but lie within a collagenous extracellular matrix. It may be that the aged retinal pigment epithelium is not able to cover the capillaries but rather changes metaplastically into fibroblasts.

Subretinal neovascularisation connected to the retinal circulation may occur in patients with idiopathic juxtafoveal telangiectasia type $2 .^{20-22}$ The surgical removal of such neovascular membranes was complicated by an intimate adherence of the membrane to the overlying neurosensory retina in an area of retinochoroidal anastomosis which supports the hypothesis that the neuroretina rather than the choroid is the primary source of the new vessels. ${ }^{23}$ Unfortunately the histopathology of the surgically removed specimens was not reported. ${ }^{23}$ Retinal vessels extending into the outer nuclear layer and subretinal space were recently described in a postmortem histopathological study of presumed juxtafoveal telangiectasia. ${ }^{24}$ Vertically oriented capillaries extended from the inner plexiform to the outer plexiform layer, some of these were continuous with subretinal vascular aggregates in intimate contact with the retinal pigment epithelium and Bruch's membrane; at one site, a continuity with the choroid was even demonstrated. ${ }^{24}$

Deep retinal vascular anomalous complexes, ${ }^{11}{ }^{13}$ also termed chorioretinal anastomoses ${ }^{1214}$ in age related macular degeneration, should not be confused with retinal participation in the vascularisation of a disciform scar which was termed retinal choroidal anastomosis. ${ }^{6910}$ In the latter complication, the disciform scar has originated from choroidal neovascularisation strictu sensu with only retinal vascular participation in the end stage. In deep retinal vascular anomalous complex, disciform scarring is not seen and the neovascular tissue does not appear to be supplied by the choroid.

In conclusion, a deep retinal vascular anomalous complex in age related macular degeneration represents histologically neovascularisation growing out of the neuroretina into the subretinal space that mimics choroidal neovascularisation. The term appears therefore rightly chosen. In some specimens fibrous tissue was found on the choroidal side of the diffuse drusen without vascular elements. Vascular anastomoses with the choroid were not observed; however, their presence could not be excluded with certainty. 
The authors thank Professor Jean-Jacques De Laey for reviewing the manuscript.

1 National Society to Prevent Blindness. Visual problems in the United States: definitions, data sources, detailed data tables, anited States: definitions, data sources, detailed data tables, analyses, interpretation. New

2 O'Shea JG. Age-related macular degeneration. Postgrad Med F 1998:74:203-7.

3 Bird AC, Bressler NM, Bressler SB, et al. An international classification and grading system for age-related maculopathy and age-related macular degeneration. Surv Ophthalmol 1995;39:367-74.

4 Vinding T. Visual impairment of age-related macular degeneration. An epidemiological study of 1000 aged individuals. Acta Ophthalmol 1990;68:162-7.

5 Sarks SH. New vessel formation beneath the retinal pigment epithelium in senile eyes. Br F Ophthalmol 1973;57:951-65.

6 Green WR, Enger C. Age-related macular degeneration histopathologic studies. The 1992 Lorenz E Zimmerman lecture. Ophthalmology 1993:100:1519-35.

7 Grossniklaus HE, Green WR, the Submacular Surgery Trials Research Group. Histopathologic and ultrastructural findings of surgically excised choroidal neovascularization. Arch Ophthalmol 1998;116:745-9.

8 Lafaut BA, Bartz-Schmidt KU, Vanden Broecke C, et al. Clinicopathological correlation in exudative age related macular degeneration: histological differentiation between classic and occult choroidal neovascularisation. $\mathrm{Br} f$ Ophthalmol, 2000;84:239-43.

9 Gass JDM. Pathogenesis of disciform detachment of the neuroepithelium. III. Senile disciform degeneration. $A m \mathcal{F}$ Ophthalmol 1967;63:617-44

10 Green WR, Gass SDM. Senile disciform degeneration of the macula; retinal arterialization of the fibrous plaque demonstrated clinically and histopathologically. Arch Ophthalmol 1971;86:487-94

11 Hartnett ME, Weiter JJ, Garsd A, et al. Classification of retinal pigment epithelial detachments associated with drusen. Graefes Arch Clin Exp Ophthalmol 1992;230:11-19.
12 Kuhn D, Meunier I, Soubrane G, et al. Imaging of choubrane G, et al. Imaging of chorioretinal anastomoses in vascularized retinal pigment

13 Hartnett ME, Weiter JJ, Staurenghi G, et al. Deep retinal vascular anomalous complexes in advanced age-related macular degeneration. Ophthalmology 1996;103:2042-53.

14 Soubrane G, Kuhn D, Quaranta M, et al. Development of retinal choroidal anastomosis in age-related macular degeneration. Invest Ophthalmol Vis Sci 1999;40(suppl): 1997.

15 Grossniklaus HE, Hutchinson AK, Capone A, et al. Clinicopathologic features of surgically excised choroidal neovascular membranes. Ophthalmology 1994;101:1099-11.

16 Caldwell RB. Blood-retinal barrier changes in the retinal pigment epithelium of RCS rats with inherited retinal degeneration. In: Hollyfield JG, Anderson RE, LaVail MM, eds. Degenerative retinal disorderds: clinical and laboratory investigations. London: Alan R Liss, 1987:333-47.

17 Bellhorn RW, Burns MS, Benjamin JV. Retinal vessel abnormalities of phototoxic retinopathy in rats. Invest Ophthalmol Vis Sci 1980;19:584-95.

18 Bellhorn RW, Bellhorn M, Friedman AH, et al. Urethaninduced retinopathy in pigmented rats. Invest Ophthalmol 1973;12:65-76

19 Korte GE, Bellhorn RW, Burns MS. Urethane-induced rat retinopathy. Plasticity of the blood-retinal barrier in disease. Invest Ophthalmol Vis Sci 1984;25:1027-34.

20 Gass JDM, Blodi BA. Idiopathic juxtafoveolar retinal telangiectasis. Update of classification and follow-up study. Ophthalmology 1993;100:1536-46.

21 Lee BL. Bilateral subretinal neovascular membrane in idiopathic juxtafoveolar telangiectasis. Retina 1996;16:344-6.

2 Park D, Schatz H, McDonald R, et al. Fibrovascular tissue in bilateral juxtafoveal telangiectasis. Arch Ophthalmol 1986; 114:1092-6.

23 Berger AS, McCuen BW, Brown GC, et al. Surgical removal of subfoveal neovascularization in idiopathic juxtafoveolar retinal telangiectasis. Retina 1997;17:94-8.

24 Eliassi-Rad B, Green WR. Histopathologic study of presumed parafoveal telangiectasis. Retina 1999;19:332-5. 